http://dx.doi.org/10.7494/automat.2012.16.2.137

Jerzy Martyna*

\title{
Runway Scheduling with Holding Pattern and Service Priority
}

\section{Introduction}

A 'Runway Scheduling Problem' may be defined as a scheduling that is able to handle a number of aircrafts allocated to landing or starting on the limited number of runways and air traffic controllers. In general, this planning problem, known as the 'Time Slot Allocation' problem has been studied in the literature (see $[2,3,6]$ ).

It is obvious that planning of airport arrivals and departures is a very complex task and plays a crucial role for each airport. Among others, it can reduce all delays while keeping the required safety standards. The typical landing planning starts when the TRACON (Terminal Radar Approach CONtrol) settles an aircraft between 5 and 50 miles of the airport. Thus, air traffic controllers make some aircraft wait before landing. Unfortunately, this 'waiting' before landing can be constrained by the circumstances, such as bad weather, lack of fuel, etc. Generally speaking, the normal reason for a hold being issued is that the airport is experiencing delays because of weather conditions, limits the capacity of the system, etc.

Therefore, some of the aircrafts are more often prioritized. To reach 'waiting' process before landing of aircrafts, two kinds of delaying procedures are used [4]:

1. Speed reduction of the aircrafts which can increase the arrival time. The aircraft speed cannot change much because it is heavily constrained by the type of aircraft, etc. For all that the speed of the aircraft can be slightly decreased to delay of the aircraft arrival.

2. 'Holding Patterns' are used to increase the arrival time of the aircrafts (see Fig. 1). Several holding patterns may be defined in the same TRACON system. By use of the holding patterns all new aircrafts enter at the top of the stack. As the bottom flight level, higher aircrafts are descended in the stack.

* Jagiellonian University, Institute of Computer Science, Prof. S. Łojasiewicza 6, 30-348 Cracow, Poland 


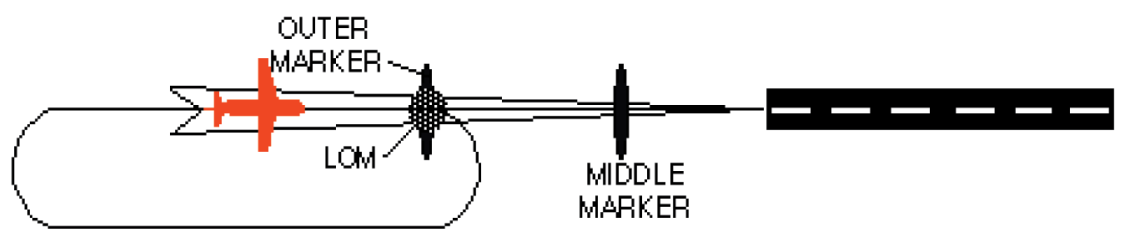

Fig. 1. Holding on a localize, with the Fix being the LOM

The problem of runway scheduling with holding patterns has first been studied by A.M. Bayen et al. [3]. In that study only a single window was used. The author's goal of optimization was to maximize the minimum distance between two consecutive landings. As was given by the authors, this problem is a special case of the decision variant of $1\left|r_{i}, p_{i}=p\right| L_{\max }$ and can be solved in polynomial time.

In the paper by A.M. Bayen et al. [5] a more general problem was studied. The goal was to minimize the total waiting time for aircrafts or to minimize the latest landing time. A scheduling problem, in which a set of aircrafts are about to land on a single runway, was proposed by K. Artiouchine et al. [1]. The objective of this study was to maximize the minimum time elapsed between any two consecutive landings. Nevertheless, none of the above mentioned papers has taken into consideration the priorities of the aid traffic landings.

The main goal of this paper is to maximize the sum of the waiting times of all aircrafts with the simultaneous consideration of landing priorities. Our problem was formulated as the mixed integer three-dimensional problem. Some experimental results are provided, in order to treat landing aircrafts at various service priorities. The rest of the paper is as follows. In the section 2 we formulate a theoretical model of the runway scheduling problem. Section 3 describes our solution of the runway scheduling with the holding patterns and service priority. In the section 4 we provide some experimental results. We finish by drawing some conclusions in section 5.

\section{Problem Formulation}

We assume that there are $\mathrm{n}$ aircrafts entering the area controlled by the TRACON system. To all aircrafts the disjoint time intervals are assigned. Each time interval corresponds to possible landing times which take into account the possible patterns and all possible speed modification. Two objective functions can be considered:

- maximizing the minimum time elapsed between any two consecutive landings,

- minimizing the maximum the number of times a plane enters a holding pattern.

Both the above described problems can be considered as a single machine scheduling in which $n$ 'landing' aircrafts with identical landing time $p$ have to be scheduled within some time windows on a single 'runway' airport. This problem can be illustrated in Figure 2. 

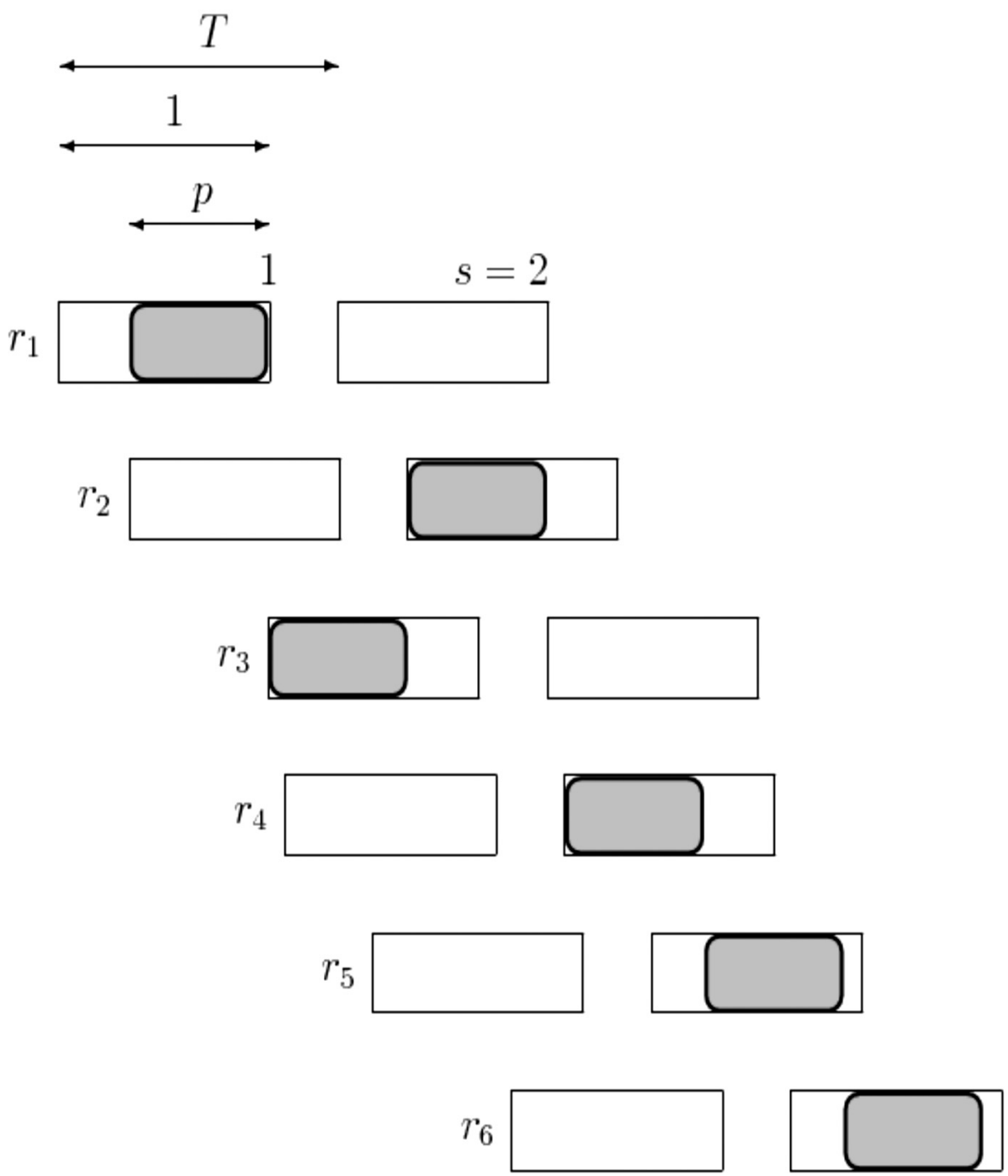

Fig. 2. The runway scheduling problem where 6 aircrafts with 2 time windows

In Figure 2 each aircraft $i$ has two windows. It has processing time $p$ in which it has to be fully scheduled (i.e. started or landed) in one of the intervals $\left[r_{i u}, d_{i u}\right]$. Every aircraft is scheduled without preemption and no two aircrafts overlap. All distances between time windows are identical. The shaded bars show a feasible solution. In Figure 2 six aircrafts with two time windows are landing on the single runway.

The runway scheduling problem in which there is a single pattern for the time windows, can be expressed in the form: the same number $s$ of windows per aircraft, the 
same window size 1 and the identical distances $T$ between all windows. This problem can be formulated as follows:

$$
\begin{aligned}
& \forall i \leq n, s_{i}=s \\
& \forall i \leq n, \forall j \leq s, d_{i j}-r_{i j}=l \\
& \forall i \leq n, \forall j \leq s, r_{i j}=r_{j}+(j-1) T
\end{aligned}
$$

\section{Formulation of the runway scheduling problem with service prio-rity}

We assume that each runway can service one aircraft at a time and that there are no technical restrictions such as the relationship between the aircraft and the runway.

Firstly, we formulate the single runway problem without the service priority with one window. We define binary variables $x_{i j k}$ to specify if aircraft $i$ is serviced (landed or star-ted) as the $k$-th aircraft at the runway with $j$ windows. As the variable $x_{i j k}$ is restricted to $0-1$ values, the runway scheduling problem may be formulated as a mixed integer three-dimensional problem as follows:

$$
\operatorname{minimize} \sum_{i \in S} \sum_{j \in V} \sum_{k \in U}\left\{(T-k+1) C_{k i}+S_{k}-A_{i}\right\} \cdot x_{i j k}+\sum_{i \in S} \sum_{j \in W_{k}} \sum_{k \in U}(T-k+1) \cdot y_{i j k}
$$

subject to

$$
\begin{gathered}
\sum_{i \in S} \sum_{k \in U} x_{i j k}=1 \quad \forall j \in V \\
\sum_{j \in V} x_{i j k} \leq 1 \forall i \in S, k \in U \\
\sum_{l \in V} \sum_{m \in P_{k}}\left(C_{k i} x_{i l m}+y_{i l m}\right)+y_{i j k}-\left(A_{i}-S_{k}\right) x_{i j k} \geq 0 \forall i \in S, j \in W_{k}, k \in U \\
x_{i j k} \in\{0,1\}, i \in S, j \in V, k \in U \\
y_{i j k} \geq 0 \quad \forall i \in S, j \in V, k \in U
\end{gathered}
$$

where $S$ is the set of aircrafts, $V$ is the set of windows orders, $U$ is the set of runways, $A_{i}$ is the arrival time of aircraft $i, P_{k}$ is the subset of runways such that $P_{k}=\{p \mid p<k \in U\}, S_{k}$ is the time when runway $k$ becomes idle for planning horizon, $W_{k}$ is the subset of aircrafts with $A_{i} \geq S_{k}, C_{k i}$ the handling time spent by aircraft $i$ at runway $k, x_{i j k}=1$ if aircraft $i$ is serviced with $j$-th windows at the runway $k$ and is equal to 0 otherwise. $y_{i j k}$ is the idle time of runway 
$k$ between the departure of the $(i-1)$-th aircraft and the arrival of $i$-th aircraft when window $j$-th is occupied in the runway $k$.

Objective function (4) minimizes the sum of waiting and service time for every aircraft. The first constraint (5) enforces that every aircraft must be serviced at the runway in any order of service. Constraints (6) ensure that the runway services up to one aircraft at any time. Constraints (7) assure that aircrafts are serviced after their arrival.

Next, we consider priority as a function of the aircraft's significance. The priority consideration is taken into account by the service time of an aircraft weighted by an incident parameter assigned to that aircraft. Thus, the aircraft's holding time contributes to its successor's service times by multiplying it by each successor's parameter.

The problem of runway scheduling with the service priority may be formulated as follows:

$$
\begin{aligned}
& \operatorname{minimize} \sum_{i \in S} \sum_{j \in V} \sum_{k \in U}\left\{C_{i j}-A_{i}+S_{k}+\sum_{l \in V} \sum_{m \in Q_{k}} C_{i l} x_{i l m}\right\} \alpha_{i} x_{i j k}+ \\
& +\sum_{i \in S} \sum_{j \in W_{i}} \sum_{k \in U}\left\{y_{i j k}+\sum_{l \in V} \sum_{m \in Q_{k}} y_{j l m}\right\} \alpha_{i}
\end{aligned}
$$

subject to

$$
\begin{gathered}
\sum_{l \in V} \sum_{m \in Q_{k}}\left(y_{i l m}+C_{i l} x_{i l m}\right)+y_{i j k}-\left(A_{i}-S_{k}\right) x_{i j k} \geq 0 \forall i \in S, j \in W_{w}, k \in U \\
\sum_{j \in V} x_{i j k} \leq 1 \quad \forall i \in S, k \in U \\
\sum_{i \in S} \sum_{k \in U} x_{i j k}=1 \quad \forall j \in V \\
x_{i j k} \in\{0,1\} \quad \forall i \in S, j \in V, k \in U \\
y_{i j k} \geq 0, \forall i \in S, k \in U, j \in V
\end{gathered}
$$

where $\alpha_{i}$ is a priority weight for aircraft $i$.

The priority weights are assigned in the following way. If the aircraft $i$ needs to be serviced at a higher priority, we can define $\alpha_{i}$ as the reciprocal of significance of aircraft $i$. It means that the most important aircraft has the highest priority which is defined by the lowest value of weight parameter. For example, when an aircraft must be quickly serviced for a certain reason, such as an emergency, high priority may be realized by adding a high value to its importance index. 
This problem can be modified by a relaxation formulation and by putting corresponding Lagrangian multipliers. Thus, this problem is as follows:

$$
\begin{aligned}
& \operatorname{minimize} \sum_{i \in S} \sum_{j \in V} \sum_{k \in U}\left\{C_{i j}-A_{i}+S_{k} \sum_{l \in V} \sum_{m \in Q_{k}} C_{i j} x_{i l m}\right\} \alpha_{i} x_{i j k}+ \\
& +\sum_{i \in S} \sum_{j \in W_{i}} \sum_{k \in U}\left\{y_{i j k}+\sum_{l \in V} \sum_{m \in Q_{k}} y_{i l m}\right\} \alpha_{i} \\
& -\sum_{i \in S} \sum_{j \in W_{i}} \sum_{k \in U} \lambda_{i j k}\left\{\sum_{l \in V} \sum_{m \in Q_{k}}\left(y_{i l m}+C_{i l} x_{i l m}\right)+y_{i j k}-\left(A_{i}-S_{k}\right) x_{i j k}\right\}
\end{aligned}
$$

subject to (12), (13), (14), and (15).

Assuming that $F_{i j k}=\left(C_{i j}-A_{i}+S_{k}\right) \alpha_{i}+\lambda_{i j k}\left(A_{i}-S_{k}\right), G_{i j k l}=\lambda_{i j k} C_{i l}, G_{i j k l}=\alpha_{i} C_{i l}$, the objective function is formulated as follows:

$$
\begin{aligned}
& \operatorname{minimize} \sum_{i \in S} \sum_{j \in V} \sum_{k \in U}\left\{F_{i j k}+\sum_{l \in V} \sum_{m \in Q_{k}} G_{i j l} x_{i l m}\right\} x_{i j k}-\sum_{i \in S} \sum_{j \in V} \sum_{k \in U} \sum_{l \in V} \sum_{m \in Q_{k}} G_{i j k l} x_{i l m} \\
& =\sum_{i \in S} \sum_{j \in V} \sum_{k \in U} F_{i j k} x_{i j k}-\sum_{i \in S} \sum_{j \in V} \sum_{k \in U}\left(\sum_{l \in V} \sum_{m \in Q_{k}} G_{i j k l}+\sum_{l \in V} \sum_{m \in Q_{k}} H_{i j l} x_{i j k}\right) x_{i l m}
\end{aligned}
$$

Now, the formulated problem is a quadratic assignment problem and it is difficult to find the optimal solution. The quadratic assignment problem can be formulated as follows:

$$
\operatorname{minimize} \sum_{j \in V} \sum_{p \in P} D_{j p} x_{j p}+\sum_{j \in V} \sum_{p \in P} \sum_{q \in Q} \sum_{n \in N} E_{j p q n} x_{n q} x_{j p}
$$

subject to

$$
\begin{aligned}
& \sum_{p \in P} x_{j p}=1 \quad \forall j \\
& \sum_{j \in V} x_{j p}=1 \quad \forall p \\
& x_{j p} \in\{0,1\} \quad \forall j, p
\end{aligned}
$$

where $q, n$ are resource (i.e. the mixture of runway and service order) indices, $P=\{S \cup U\}$, $Q=\{S \cup V\}$.

Due to the NP-hardness [9], the quadratic assignment problem can be solved not only exactly but also approximately. Among others, constrained neural approaches to quadratic assignment problems and the efficient genetic algorithms has been proposed by $\mathrm{S}$. Ishii et al. [7] and M.H. Lim et al. [8], respectively. 


\section{Numerical Experiments}

To one of the exactly solution so formulated problem, we can use the simplex method. The solution program was written in Matlab language. We have developed two problems in which 20 and 50 aircrafts are landed without and with priorities. Both data sets are prepared for one runway airport with the possible various holding time. The planning horizons for the first and the second set of aircrafts are equal to 5 and 10 minutes, respectively.

Figure 3 shows the average holding time of the aircrafts from the data sets A and B without and with the service priority and for the four runways and horizon time equal to 5 minutes, respectively. The data set of the aircrafts without the service priority have the mean holding time longer than the data set of aircrafts with priority. This results occurs because in such cases the aircrafts do not come very often.

a)

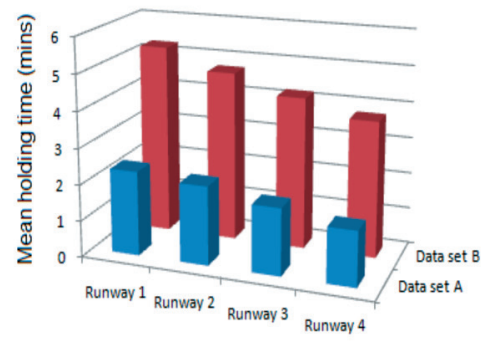

b)

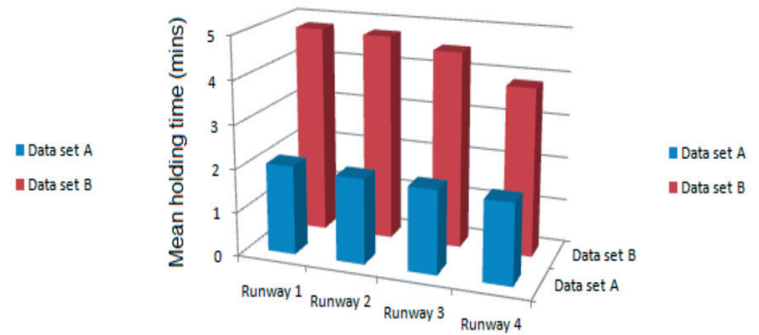

Fig. 3. Mean holding time of the aircrafts for four runways and both data sets without (a) and with the service priorities (b). Planning horizon is equal to 5 minutes

Figure 4 presents the average value of the holding time of aircrafts from the data sets A and $\mathrm{B}$ without and with the service priority for the four runways and horizon time equal to 10 minutes, respectively. Analogically as in the previous case, the data set of aircrafts with the priority service has a shorter mean holding times than the same data set without service priority.

a)

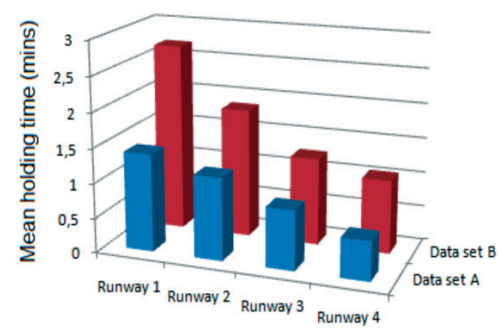

b)

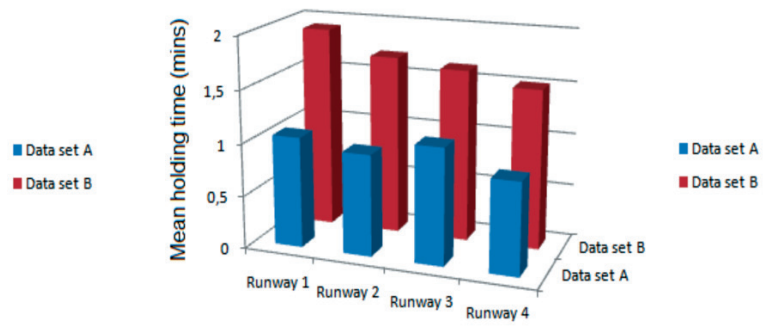

Fig. 4. Mean holding time of the aircrafts for four runways and both data sets without (a) and with the service priorities (b). Planning horizon is equal to 10 minutes 


\section{Conclusion}

In this study, we examined the priority service in the runway sequencing with holding patterns. We have formulated this scheduling problem as a mixed integer three-dimensional problem. As an effective solution of this problem, we have eventually employed a heuristic algorithm, which allows us to obtain results in a relatively shorter time comparing with the numerical methods. A number of computational experiments were conducted showing the priority service.

In the future work we will use the genetic algorithms and the SVM methods in the minimization of waiting time of aircrafts before landing. Also, the extensive simulation of runway sequencing will be helpful in finding the best scheduling for the airport traffic.

\section{References}

[1] Artiouchine K., Baptiste Ph., Ch. Dürr Ch., Runway Sequencing with Holding Patterns. European Journal of Operational Research, vol. 189, No. 3, 2008, 1254-1266.

[2] Barnier N., Brisset P., Slot Allocation in Air Traffic Flow Management. http://www.recherche.enac.fr/opti/.

[3] Bayen A.M., Tomkin C.J., Ye Y., Zhang J., MILP Formulation and Polynomial Time Algorithm for an Aircraft Scheduling Problem. http://cherokee.stanford.edu/ bayen/publications.html.

[4] Bayen A.M., Tomkin C.J., Real-Time Discrete Control Law Synthesis for Hybrid System Using MILP: Application to Congested Airspace. Proc. of the American Control Conference, 2003.

[5] Bayen A.M., Tomlin C.J., Ye Y., Zhang J., An Approximation Algorithm for Scheduling Aircraft with Holding Time. Proc. of the 43rd IEEE Conference on Decision and Control, 2004.

[6] Brücker P., Scheduling Algorithms. 2 ed., Springer, Heidelberg, 1998.

[7] Ishii S., Sato M., Constrained Neural Approaches to Quadratic Assignment Problem. Neural Networks, vol. 11, 1998, 1073-1082.

[8] Lim M.H., Yuan Y., Omatu S., Efficient Genetic Algorithms Using Simple Gene Exchange Local Search Policy for the Quadratic Assignment Problem. Computational Optimization and Applications, vol. 15, 2000, 249-268.

[9] Pinedo M., Scheduling: Theory, Algorithms and Systems. 3 ed., Springer, Heidelberg, 2008. 